Original Research

\title{
Rider and Horse Salivary Cortisol Levels During Competition and Impact on Performance
}

\author{
Marie Peeters MSc, Coline Closson MSc, Jean-François Beckers PhD, Marc Vandenheede PhD \\ Faculty of Veterinary Medicine, University of Liège, Liège, Belgium
}

\section{A R T I C L E I N F O}

\section{Article history:}

Received 22 August 2011

Received in revised form

29 September 2011

Accepted 31 May 2012

Available online 3 August 2012

\section{Keywords:}

Stress

Competition

Cortisol

Riders

Horses

\begin{abstract}
A B S T R A C T
During competition, stress may affect riders and horses. This stress can affect health, welfare, and/or performance. Our aim was to quantify stress levels during competition in horses and riders. We also searched relationships between these stress levels and performance. Twenty riders and 23 horses were followed up during a show-jumping event ( 26 courses) held at a riding school. Regular saliva samples taken from horses and riders were assayed to evaluate cortisol levels. We studied salivary cortisol evolution during the days of competition. There was no correlation between instantaneous sampling on horses and their riders. However, we did find a parallel between horse and rider salivary cortisol evolution curves, with a similar peak, reached 20 minutes after the course. The increase was stronger in riders than in horses. Correlations appeared between salivary cortisol concentration and performance, but stress in both partners seems to have an opposite influence on performance. Riders who showed a higher salivary cortisol increase were awarded more penalties, whereas horses that showed a higher increase in salivary cortisol performed better. Stress level measurement in rider-horse pairs would thus lead to improvement in competition conditions and performance, for horses as well as for riders.
\end{abstract}

(c) 2013 Elsevier Inc. All rights reserved.

\section{Introduction}

In every sport, competition induces stress. This also occurs in equestrian sports. Riders and horses can be affected by stress at various levels. Stress is not always harmful. Positive stress, called "eustress," is even necessary for environmental adaptation. It is thus important for raising resources, for example, to perform in competition. Stress may become negative when it causes deleterious effects on health, welfare, and/or performance. Stress is then called "distress." Distress can appear when an individual is confronted with an acute or a chronic stressor, which surpasses his adaptation capacities [1].

Corresponding author at: Marie Peeters, MSc, B43, Ethologie Vétérinaire te bien-être des animaux, Faculty of Veterinary Medicine, University of Liège, 20, Boulevard de Colonster, 4000 Liège, Belgium.

E-mail address: mariepeeters007@gmail.com (M. Peeters).
Competition is a mixture of various stressors (stabling, transport, novelty, rider stress, noisy public, and music). Nevertheless, only a few stress studies have been carried out in equestrian sports. One of them examined the effects of show-jumping competition on horse stress levels. Researchers took blood samples at the horse show and at home for plasma cortisol assessment. They found that competition experience had an effect on physiological stress: less experienced horses displayed higher plasma cortisol values when at rest at the show than at home [2]. Another study examined the effect of show-jumping as well as dressage on stress levels by comparing horses' stress response at a horse show and at home. They found that competition induced a significant increase in blood cortisol response in both jumping and dressage horses. They conclude that competition elicits a classic physiological stress response in horses [3].

Other researchers measured plasma cortisol concentrations before and after show-jumping competitions. 
Horses were separated, according to their competition experience and if they were transported or not just before competition. They found significant increases relative to the basal values in cortisol concentrations of all groups of horses [4]. In a preliminary study, blood samples were taken before and after an international cross event. Results showed that serum cortisol concentrations were higher after than before the cross event [5].

As largely accepted, saliva sampling is a noninvasive alternative technique for assessment of cortisol levels in horses. This method is more appropriate for physiological stress assessment in horses, even more so in competition. Saliva sampling can be performed anywhere, by every horseman on every domesticated horse, after only a short period of training. Sampling is easy and stress and pain free [6]. Therefore, we preferred to collect saliva than blood to measure the activity of the hypothalamo-pituitary-adrenal axis (HPAA). This salivary corticosteroid is largely used as a stress hormone in humans [7] as well as in horses [5,8,9]. A cortisol concentration increase is observed in saliva with a delay of approximately 20-30 minutes before it is observed in blood. This has been observed in horses $[6,10]$ as well as in humans [11].

Saliva cortisol concentrations have already been used in stress studies involving horses exposed to stressors such as a new environment [12], transport [9,13], and competition [5]. Horses' salivary cortisol basal values differed largely between individuals [5]. It was recommended to measure basal values for each individual before measuring concentrations during stress events [13].

Salivary cortisol is frequently used as a biomarker in human stress research [14]. As in horses, it reflects HPAA activity. Salivary cortisol level can express stress, but it can also be affected by different factors, such as age, gender, oral contraceptive, or medical conditions. These variables can affect cortisol binding and HPAA response [14]. To be able to relate salivary cortisol level with stress, we need to control for these variables as well as possible.

Basal values for salivary cortisol in humans are well described in the literature [7,15]. Daily cortisol secretion can be divided into two distinct phases: the cortisol awaking response and a subsequent period of decline throughout the rest of the day (Edwards et al. [2001] in [15]). Oskis et al. found a cortisol awakening response and a subsequent daytime decline period in female adolescents [15]. The mean ( \pm standard deviation) cortisol concentration of four saliva samples collected from 3 to 12 hours after awakening was $4.17 \pm 2.05 \mathrm{nmol} / \mathrm{L}$ [15].

The essence of the competition model is that to win, the competitor must out-stress the opponent [16]. Stress, measured by cortisol, is important for the preparation of the competition, during and after the stressful situation. Most studies detect an anticipatory rise in cortisol when they collect pre-event samples (15-30 minutes before the competition) [16]. Increasing cortisol level might be important in preparing for mental and physiological demands, and might affect performance. A moderate rise in cortisol helps individuals deal with challenges in competition (Stansbury and Gunnar [1994] in [16]). "First, it marshals resources needed for physical activity. Second, it positively affects memory, learning and emotion and thirdly, cortisol serves a homeostatic function by regulating other stress sensitive systems" [16]. Some studies have found that extreme elevations in cortisol lead to poor performance (Erickson et al. [2003] and Cumming et al. [1983] in [16]). Kivlighan et al. [16] found that endocrine response to stress varied by gender and by experience of competition. Responses were also different depending on when samples are taken in anticipation of, in response to, or after the competitive event. Another study, performed on judoists, shows an anticipatory rise in serum cortisol [17]. They also found significantly higher serum cortisol levels in winners in comparison with losers.

The neuroendocrine response to competition is thus complex and depends on subjective factors related to the cognitive evaluation of the situation rather than on the outcome itself [18]. Exercise leads to an increase in cortisol, in humans as well as in horses [19-24]. However, by taking saliva samples during a board game competition in Japan, researchers found that changes in salivary cortisol after competition are associated with winning and losing, even if this game does not need physical exercise [25]. Some studies have found that salivary cortisol is increased in current smokers, compared with nonsmokers [26]. Other studies also found that smoking a cigarette activates the HPAA in habitual smokers [27,28].

Our aims were to quantify stress levels in competition using salivary cortisol levels, following their kinetics in horses and riders, and comparing these stress levels with the performances achieved.

\section{Material and Methods}

In the present study, we tried to standardize external factors that could influence rider and horse stress. We observed horses and riders at the same place, during the same competition, and with the same experimental protocol.

We observed 20 riders and 23 Warmblood horses before and during a jumping event, organized by a riding school. The riders' school is the "Ecole provinciale d'élevage et d'équitation de Gesves" (EPEEG) in Belgium. We followed 26 jump courses spread over two consecutive days. The six riders and three horses followed twice were observed on two different days.

Riders were young women between 17 and 20 years of age, all students at this school. Participants were thus a relatively homogeneous set of female adolescents; they lived 5 days a week at the school, where they followed the same daily routine (horse riding training and classroom lessons). They had ridden $6 \mathrm{~d} / \mathrm{wk}$ for at least 2 years. They all have previous competition experience. They were also all postmenarcheal. Four of them (20\%) were regular smokers. All participants provided written informed consent.

Horses were 12 mares and 11 geldings whose ages ranged from 6 to 11 years. Horses had all been stabled at the school for at least 1 year. They had been trained $6 \mathrm{~d} / \mathrm{wk}$ (jumping and dressage lessons) for at least 2 years. Riders were the horses' owners, and pairs were used to working together.

Pairs performed over fences between 90 and $110 \mathrm{~cm}$ in height. During the 2 days of competition, we took saliva samples from riders and horses, following accurate timing: 
before warm-up (at $9 \mathrm{Am}$ ), just before entering the competition ring, and 20,40, and 60 minutes after the competition.

Saliva was sampled using Salivettes (Sarstedt, Nümbrecht, Germany). A swab on a metal clamp was maintained in the horse's mouth for 30-40 seconds, above and below the tongue and placed in Salivettes. Riders could choose between chewing the swab or directly spitting saliva into the Salivette.

Salivary samples were stored at $4^{\circ} \mathrm{C}$. After the 2 days, Salivettes were centrifuged for 10 minutes at $1,500 \times \mathrm{g}$. Saliva samples were stored at $-20^{\circ} \mathrm{C}$ until assayed. Cortisol concentrations were measured by radioimmunoassay, as previously described [6]. All samples were assayed in four replicates. Means of these replicates were used in the analysis. To minimize variation, all samples from one rider or from one horse were tested in the same assay batch.

We took four salivary samples from horses during a rest morning at the school, as suggested in the article by Schmidt et al. [9]. Mean salivary cortisol obtained with these four samples was used as the individual horse basal salivary cortisol concentration (SCC). These four samples could not be taken from one horse (away from the school because of injury). This horse was included in showjumping measurements but excluded from baseline measurement.

Performances were scored by penalty addition during the jump course. Penalties were given by jump competition judges.

Statistical analysis was performed using correlation analysis. Spearman correlation $(P<.05)$ was chosen because most variables did not follow a normal distribution. Student $t$ test (paired data) was used to compare SCC means.

\section{Results}

\subsection{SCC Evolution in Horses}

We sampled 23 horses during competition. Three of these horses were sampled during two jump courses, on two successive days. So, we took saliva samples during 26 jump courses (126 saliva samples). Four samples were missing, owing to sampling problems (not enough saliva in the Salivette) or difficulties to find the horse after competition (walking outside). Horses' mean SCCs are presented in Table 1.

Morning SCCs did not differ significantly from baseline SCCs (paired $t$ test; $P=.20$ ).

During the competition, SCC increased from the morning sample to 20 minutes after the start of the jump course, and then decreased until 60 minutes after the start of the jump course. Values at 20 minutes after start of jump

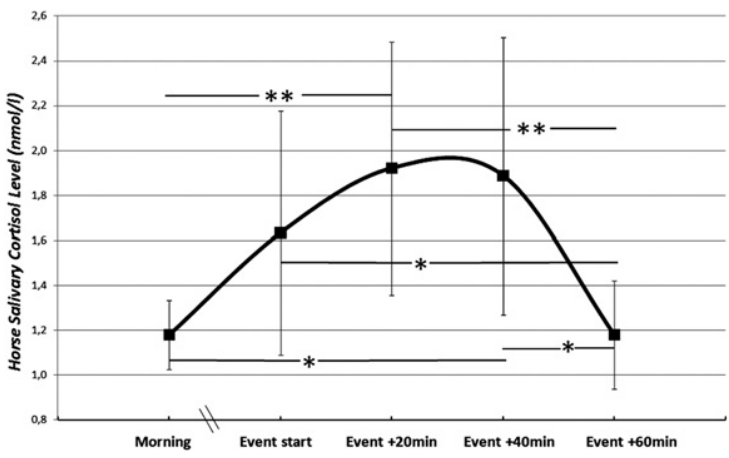

Fig. 1. Mean horse salivary cortisol level evolution in competition. ${ }^{*} P<.05$; ${ }^{* *} P<.01$.

course were higher than morning SCC (paired $t$ test; $P=$ .009). SCC was still higher after 40 minutes (paired $t$ test; $P=.03$ ), and it returned to SCC morning levels 60 minutes after the beginning of the jump course (paired $t$ test; $P=$ .88). This reversed " $U$ " curve is shown in Figure 1. Mean SCC during the whole day of jumping was significantly higher than baseline (paired $t$ test; $P=.002$ ).

SCC measured at the course start was positively correlated to all-day mean SCC $\left(r_{\mathrm{S}}=0.69\right)$ and to SCC measured 20 minutes $\left(r_{\mathrm{s}}=0.70\right), 40$ minutes $\left(r_{\mathrm{s}}=0.50\right)$, and 60 minutes $\left(r_{\mathrm{s}}=0.55\right)$ after the start of jumping (Spearman correlation; $P<.05)$. SCC measured at rest was only correlated to SCC measured 40 minutes after the start of jumping $\left(r_{\mathrm{s}}=0.50\right)$.

We also did not find any difference between geldings and mares in SCC at rest and during competition ( $t$ test; $P<.05)$.

\subsection{SCC Evolution in Riders during Competition}

Mean rider SCCs are reported in Table 2. A morning sample was taken at least 3 hours after waking time in our study. Morning SCC agreed with "post-menarcheal adolescent female" SCC levels found by Oskis et al. [15] (4.17 \pm 2.05 $\mathrm{nmol} / \mathrm{L})$, which were calculated on four samples collected from 3 hours to 12 hours after awakening. The increase between morning SCC and +20 minutes SCC was faster and stronger (approximately 230\%) than observed in horses (approximately 60\%) ( $t$ test; $P<.001$ ).

Mean SCC for the day of competition was correlated to the start of the jump course $\left(r_{\mathrm{s}}=0.75\right),+20$ minutes $\left(r_{\mathrm{s}}=\right.$ $0.92),+40$ minutes $\left(r_{\mathrm{s}}=0.89\right)$, and +60 minutes $\left(r_{\mathrm{s}}=\right.$ $0.79)$ SCC.

Riders who smoke ( $\mathrm{n}=4$, five jump courses) showed a significantly higher SCC than those who do not $(n=16$, 21 jump courses) at +20 minutes (unpaired $t$ test; $P=.005$ ),

Table 1

Mean SCCs of horses at rest and during show-jumping

\begin{tabular}{|c|c|c|c|c|c|c|c|}
\hline Horse Saliva Sampling & Baseline & Jumping Day & & & & & \\
\hline Timing & At Rest & Whole Day of Competition & Morning & Event Start & +20 Minutes & +40 Minutes & +60 Minutes \\
\hline Number of observations & 22 horses & 23 horses and 26 jump courses & & & & & \\
\hline Number of saliva samples & 88 & 126 & 26 & 26 & 24 & 26 & 24 \\
\hline Mean SCC \pm SD $(\mathrm{nmol} / \mathrm{L})$ & $1.01 \pm 0.62$ & $1.56 \pm 1.18$ & $1.18 \pm 0.38$ & $1.63 \pm 1.34$ & $1.92 \pm 1.33$ & $1.53 \pm 0.62$ & $1.18 \pm 0.57$ \\
\hline
\end{tabular}

SCC, salivary cortisol concentration. 
Table 2

Mean SCCs of riders during show jumping

\begin{tabular}{|c|c|c|c|c|c|c|}
\hline Rider Saliva Sampling & Jumping Day & & & & & \\
\hline Timing & Whole Day of Competition & Morning & Event Start & +20 Minutes & +40 Minutes & +60 Minutes \\
\hline Number of observations & 20 riders and 26 jump courses & & & & & \\
\hline Number of saliva samples & 120 & 26 & 26 & 23 & 21 & 24 \\
\hline Mean SCC \pm SD $(\mathrm{nmol} / \mathrm{L})$ & $8.28 \pm 4.99$ & $5.51 \pm 2.34$ & $7.43 \pm 3.61$ & $12.64 \pm 6.39$ & $9.33 \pm 5.50$ & $7.10 \pm 3.06$ \\
\hline
\end{tabular}

+40 minutes $(P=.002)$, and +60 minutes $(P=.038)$, as well as for mean SCC during the whole day of jumping $(P=.002)$. But, they do not necessarily have more penalties $(P=.099)$ during jumping competition.

\subsection{Relationship between Rider SCC and Horse SCC Discharges}

Figure 2 shows the parallels between mean SCC kinetics of riders and horses. Horses' mean SCCs were strongly correlated to riders' mean SCCs during show jumping (Spearman correlation; $r_{\mathrm{s}}=0.96$ ). However, we found no significant correlation between SCCs of each rider and her own horse.

\subsection{Relationship between SCC and Performances}

The number of penalties was positively correlated to riders' mean SCC at peak $\left(r_{\mathrm{s}}=0.42\right)$, after 40 minutes $\left(r_{\mathrm{s}}=\right.$ $0.57)$, and after 60 minutes $\left(r_{\mathrm{s}}=0.58\right)$. On the contrary, the number of penalties was negatively correlated to mean horse SCC at peak $\left(r_{\mathrm{s}}=-0.44\right)$.

\section{Discussion}

\subsection{Evolution in Horses}

Horses' morning samples did not differ from baselines. This indicates that there was no anticipation by the horse. The fact that it is a competition performed at home could explain this absence of the horses' cortisol rise. The baseline values found in this study agree with those reported in previous studies $[5,6,13,29]$.

Mean SCCs found during competition were difficult to compare between studies because sampling protocols differ. However, the peak we observed was not high compared with increases found in other stress studies $[5,9,13]$. Our SCC peak measured during competition was approximately $190 \%$ of baseline and $160 \%$ compared with morning samples. In a preliminary study, Peeters et al. [5] found that mean SCC after cross-country events were more than three times higher (340\%) than just before starting, and in another previous study, they found a rise of $360 \%$ for horses' mean SCC during competition compared with SCC when they were at home. Schmidt et al. [13] found an increase of approximately 600\% after a trailer trip. In the present study, horses' HPAA seemed not to be stimulated as much as it has been stimulated during other competitions. Nevertheless, the results of Covalesky et al. suggested that conditioned jumpers that have previously been exposed to horse show environments do not appear stressed during acute show-jumping competitions [2]. In our study, the show jumping was organized in a familiar environment and horses were not transported.

The fact that cortisol levels at the start of the course were positively correlated to different other-time sampling after the start of jumping means that we could take only some samples from around the area of competition to assess HPAA activity.

\subsection{Evolution in Riders during Competition}

Correlations between SCC sampled at the start of jump course and after $+20,+40$, and +60 minutes allow us to recommend future studies to take only one or two saliva samples approximately 20 minutes (peak time) after the start of the jump course to assess rider stress.

The effect of smoking on cortisol levels agrees with previous findings [26-28].

\subsection{Relationship between Rider SCC and Horse SCC Discharges}

Regrettably, we found no significant correlation between SCCs of each rider and her own horse. We still assume that rider's stress level could influence horse's stress and vice versa. We suggest continuing to observe these cortisol levels simultaneously in the horse and the rider for future studies. It would be interesting to test this relation in more professional riders, competing at higher levels of show jumping.

\subsection{Relationship between SCC and Performances}

Correlations between penalties and rider SCC are "moderate" and have been reported to lead to "substantial relationships" [30]. A high rider stress level seems to be

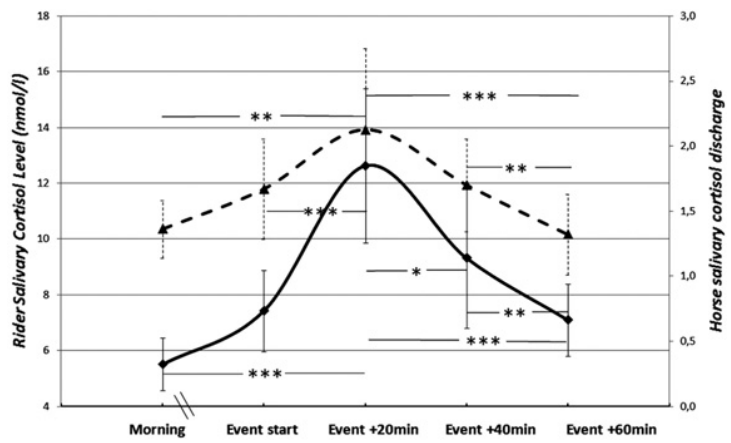

Fig. 2. Mean rider salivary cortisol levels and hiorse salivary cortisol discharge evolution. -----, mean rider SCC (nmol/L); ------, mean discharge of horse SCC. ${ }^{*} P<.05 ;{ }^{* *} P<.01 ;{ }^{* * *} P<.001$. SCC, salivary cortisol concentration. 
linked to poor performances. This stress can arise from apprehension toward competition, but it can also be maintained (+60 minutes) by bad results obtained. Compared with riders, horse stress seems to lead to better results. However, we have to remember that horses' SCC increase (160\%) was low compared with riders' SCC increase (230\%). Horses' SCC increase observed in this study was lower than that observed in other stress studies, such as transport studies. The stress induced during this competition (without any transport or any change in stabling) may be lower than the limit where stress should become distress (see Introduction), negatively influencing performance.

\section{Conclusion}

It is now possible to easily measure stress levels in horse and rider couple during competition, by assessing HPAA activity through cortisol measurement. Validation of this stress-free method, saliva sampling, in show jumping was also important. We showed that horse and rider free cortisol increases at the same time, the increase being stronger in riders than in horses. However, no correlation between the stress levels of the rider and her own horse was found. Stress of both partners seems to have an opposite influence on performance during competition. Riders who showed a higher salivary cortisol increase got more penalties, whereas horses that showed a higher salivary cortisol increase performed better. These observations were made during an internal competition, in a well-known environment, requiring no transport. According to the hypothesis that the stress/performance relationship follows the inverted U-shaped curve, we suggest the possibility that during this home competition, horses were on the left-hand side of the curve (more stress leading to enhanced performance) and riders on the right-hand side (more stress leading to reduced performance).

These results need to be confirmed by future studies, especially in an unfamiliar competition environment. There also needs to be studies of stress during sporting events of longer duration, in which the relationship between horse and rider is longer in time, as is the case of endurance. Studying stress levels in equestrian sports will lead to improving the performance and welfare of both riders and horses.

\section{Acknowledgments}

The authors thank Mrs. Duchêne for her cordial welcome at the riding school "Ecole Provinciale d'Elevage et d'Equitation de Gesves" (EPEEG, Belgium). They thank riders and horses for their good swab chewing. They are also grateful to the Reproductive Physiology Department of the University of Liège (Belgium) and the Native English reviewer Andrew Clark.

The work has been supported by grants from the Le Fonds pour la formation à la Recherche dans l'Industrie et dans l'Agriculture (FRIA)-Le Fonds de la Recherche Scientifique (Belgium) (FNRS), without any other involvements.

\section{References}

[1] Moberg GP. Biological response to stress: implications for animal welfare. In: Moberg GP, Mench JA, editors. The biology of animal stress. Wallingford: CAB International; 2000. p. 1-21.

[2] Covalesky ME, Russoniello CR, Malinowski K. Effects of showjumping performance stress on plasma cortisol and lactate concentrations and heart rate and behavior in horses. J Equine Vet Sci 1992;12:244-51.

[3] Cayado P, Munoz-Escassi B, Dominguez C, Manley W, Olabarri B, Sanchez de la Muela $\mathrm{M}$, et al. Hormone response to training and competition in athletic horses. Equine Vet J Suppl 2006;36:274-8.

[4] Fazio E, Medica P, Cravana C, Ferlazzo A. Effects of competition experience and transportation on the adrenocortical and thyroid responses of horses. Vet Rec 2008;163:713-6.

[5] Peeters M, Sulon J, Serteyn D, Vandenheede M. Assessment of stress level in horses during competition using salivary cortisol: preliminary studies. J Vet Behav 2010;5:216.

[6] Peeters M, Sulon J, Beckers JF, Ledoux D, Vandenheede M. Comparison between blood serum and salivary cortisol concentrations in horses using an adrenocorticotropic hormone challenge. Equine Vet J 2011;43:487-93.

[7] Kelly SJ, Young R, Sweeting H, Fischer JE, West P. Levels and confounders of morning cortisol collected from adolescents in a naturalistic (school) setting. Psychoneuroendocrinology 2008;33: 1257-68.

[8] Creighton E, Hughes T, Coleman R. Validation of salivary cortisol as an indicator of stress in horses (Equus caballus). In: Proceeding of the 38th International Congress of the ISAE. Col. University of Helsinki, Finland; 2004:186.

[9] Schmidt A, Möstl E, Wehnert C, Aurich J, Müller J, Aurich C. Cortisol release and heart rate variability in horses during road transport. Horm Behav 2010;57:209-15.

[10] Hughes T, Creighton E, Coleman R. Validation of salivary cortisol as an indicator of HPA activity in horses. In: Proceedings of the 41st International Congress of the ISAE. Merida, Mexico; 2006.

[11] Kirschbaum C, Hellhammer DH. Salivary cortisol in psychobiological research. An overview. Neuropsychobiology 1989;22: 150-69.

[12] Peeters M, Péters F, Sulon J, Sandersen C, Poncin P, Serteyn D, et al. Behavioural and physiological assessment of stress level in hospitalised horses: correlation between parameters. In: The 42nd Congress of the International Society for Applied Ethology. University College Dublin, Ireland; 2008.

[13] Schmidt A, Biau S, Möstl E, Becker-Birck M, Morillon B, Aurich J, et al. Changes in cortisol release and heart rate variability in sport horses during long-distance road transport. Domestic Anim Endocrinol 2010;38:179-89.

[14] Hellhammer DH, Wüst S, Kudielka BM. Salivary cortisol as a biomarker in stress research. Psychoneuroendocrinology 2009;34: 163-71.

[15] Oskis A, Loveday C, Hucklebridge F, Thorn L, Clow A. Diurnal patterns of salivary cortisol across the adolescent period in healthy females. Psychoneuroendocrinology 2009;34:307-16.

[16] Kivlighan KT, Granger DA, Booth A. Gender differences in testosterone and cortisol response to competition. Psychoneuroendocrinology 2005;30:58-71.

[17] Suay F, Salvador A, González-Bono E, Sanchís C, Martínez M, Martínez-Sanchis S, et al. Effects of competition and its outcome on serum testosterone, cortisol and prolactin. Psychoneuroendocrinology 1999;24:551-66.

[18] Salvador A. Coping with competitive situations in humans. Neurosci Biobehav Rev 2005;29:195-205.

[19] Art T, Lekeux P. Physiological responses to extreme heat and relative humidity-studies on the effects of exercise. Pratique Vet Equine 1996;28:3-8.

[20] Colborn DR, Thompson DL Jr, Rahmanian MS, Roth TL. Plasma concentrations of cortisol, prolactin, luteinizing hormone, and follicle-stimulating hormone in stallions after physical exercise and injection of secretagogue before and after sulpiride treatment in winter. J Anim Sci 1991;69:3724-32.

[21] Colborn DR, Thompson DL Jr, Roth TL, Capehart JS, White KL. Responses of cortisol and prolactin to sexual excitement and stress in stallions and geldings. J Anim Sci 1991;69:2556-62.

[22] Gordon ME, McKeever KH, Betros CL, Manso Filho HC. Exerciseinduced alterations in plasma concentrations of ghrelin, adiponectin, leptin, glucose, insulin, and cortisol in horses. Vet J 2007;173:532-40.

[23] Malinowski K, Shock EJ, Rochelle P, Kearns CF, Guirnalda PD, McKeever KH. Plasma beta-endorphin, cortisol and immune 
responses to acute exercise are altered by age and exercise training in horses. Equine Vet J Suppl 2006;36:267-73.

[24] Marc M, Parvizi N, Ellendorff F, Kallweit E, Elsaesser F. Plasma cortisol and ACTH concentrations in the warmblood horse in response to a standardized treadmill exercise test as physiological markers for evaluation of training status. J Anim Sci 2000;78:1936-46.

[25] Hasegawa M, Toda M, Morimoto K. Changes in salivary physiological stress markers associated with winning and losing. Biomed Res 2008;29:43-6.

[26] Badrick E, Kirschbaum C, Kumari M. The relationship between smoking status and cortisol secretion. J Clin Endocrinol Metab 2007;92:819-24.
[27] Rohleder N, Kirschbaum C. The hypothalamic-pituitary-adrenal (HPA) axis in habitual smokers. Int J Psychophysiol 2006;59: 236-43.

[28] Kirschbaum C, Wüst S, Strasburger CJ. 'Normal' cigarette smoking increases free cortisol in habitual smokers. Life Sci 1992;50: 435-42.

[29] Moons CPH, Laughlin K, Zanella AJ. Effects of short-term maternal separations on weaning stress in foals. Appl Anim Behav Sci 2005;91:321-35.

[30] Martin P, Bateson P. Measuring behaviour-an introductory guide. In: Paul Martin, Patrick Bateson, editors. 2nd ed. Cambridge: Cambridge University Press; 1993. 